\title{
Laser Based Real Time Antenna Pointing Measurement System
}

\author{
Anish Kumar ${ }^{1}$, Thangadurai $\mathbf{N}^{2 *}$ \\ ${ }^{I} P G$ Scholar, Department of Electronics Communication and Engineering \\ ${ }^{2}$ Associate Professor and HOD, Department of Electronics Communication and Engineering \\ School of Engineering and Technology, Jain University, Bangalore, India \\ *Corresponding Author Email: ${ }^{2}$ mrgoldjain2015@gmail.com
}

\begin{abstract}
The antenna pointing system is very important to obtain an optimum received signal level. To achieve it, presenting the implication of laser beam radiated emission requirements and how they state for pointing measurement system, its impact on design, verification and testing for the antenna pointing system. The antenna pointing system is developed by using array of linear light sensors, crosshair laser weapon, where the light sensors are mounted on the board and laser weapon putted on the antenna mechanism. On the rotator system, composed by using 2 gears and 2 DC motors with customized frames. For collecting the sensors data using ProASIC3 FPGA fabric and finding the numbers and which sensors has been active during the emitting period and 32-bit ARM Cortex-M3 processor take account into the pointing measurement system to computing the pointing and pointing error. At the end, the final step is building a User Graphical Interface on PC to show the output of the antenna pointing.
\end{abstract}

Keywords: Linear Pixel Sensor, Antenna Mechanism, Crosshair laser, 32-bit ARM processor

\section{Introduction}

Pointing measurement system include cross laser module transmitter mounted on Dual Gimbal Antenna (DGA) mechanism, which drives the antenna on- board a satellite to track the desired ground station in real time as the satellite is maneuvered at a stationary reference position and a receiver consist array of pixel sensor at mobile site. The transmitter produced laser reference plane by sweeping a laser beam about the transmitter. The receiver includes array of pixel sensor when the laser beam is impinging on the pixel of sensor generate photo current which amplify by the voltage amplifier at the end of the sensor and accounting a development board called Smart Fusion development board consist of fusion of ProASIC FPGA Fabric operated at $350 \mathrm{MHz}$ and 32-bit ARM Cortex®-M3 Processor with 100MHZ CPU speed and Analog compute engine and The system includes a circuit composed of a photo detector, timer, clock and counter. Generated analog signal is collected by the sensor processing electronic PCB which is interconnected between sensor board and development board, sensor processing PCB collect all analog data from the sensor board forward to development board in the analog form, development board is sampled the analog signal at 0.5 us interval through this sample vale is find the intercepting point on the sensor board and obtain the pointing and accuracy of antenna. At the end of these displaying the output of the pointing on the PC by GUI interface which provide the accuracy vale and pixel value of sensors.

\section{Literature Survey}

The antenna pointing is very important to obtain an optimum signal received and in order to track the satellite with the proper accuracy. Many research papers and scholarly articles have been written and they have proposed the unique and innovative method

to improve the pointing measurement system in antenna pointing system.

Alfonso Muñoz Hernandez, Jose Gala Escolar, Jose Fernandez Pardo, (2017) are implement the antenna pointing subsystem by low frequency radiated emission from E-field and $\mathrm{H}$ - field. It composed antenna pointing mechanism (APM) which is part of this subsystem, composed by structure to support to antenna and composed by some motor and position sensors to provide required direction.Also describe the ground design implementation composed by antenna pointing mechanism electronics (APME) to provide the power to motor according to command received and position information read from the sensors and provide to reduce the radiated emission generated inside of subsystem.It is limited for the radiated emission for E-field and $\mathrm{H}$-field at low frequency and designing with this new limit is more challenging and more difficult.

Rama Setya Anggara, (2014) describe about the automatic antenna pointing and using three frequenciesareS-band frequency; also VHF and UHF band those use three different antenna for remote sensing and rotator system consist 2 gears and 2 DC motor which provide required direction of antenna.

Automatic antenna pointing done by compass antenna CPMS10 as a feedback and 16-bit process, microcontroller calculate angle of tracked satellite to provide the voltage to rotator at 7.3 degree per second azimuth speed and 7.7 degree per second elevation speed on average.It provides the reduce the miss pointing but the loadtest show that on average 98 kilograms load for azimuth movement needs 58.8 Watts supply; and 54 kilograms-load for the elevation movement needs 198 Watts supplies. Antenna pointing give 1 degree accuracy wit maximum error of 0.5 degree. 
H. Yu, (2017) is investigation about the antenna pointing model and try to obtain high accuracy of pointing by reducing the pointing error, this investigation focus on $\mathrm{X}$ - band frequency, it build the error correction model including Gaussian and nonlinear term.Error correction model required whole sky-data in azimuth and elevations, this data is obtain by cross scan the radio source, collected data fitting by Gaussian and calculating the pointing error.Pointing accuracy meet the requirement of the 15 arcsec pointing accuracy at the $\mathrm{X}$ band at low cost.

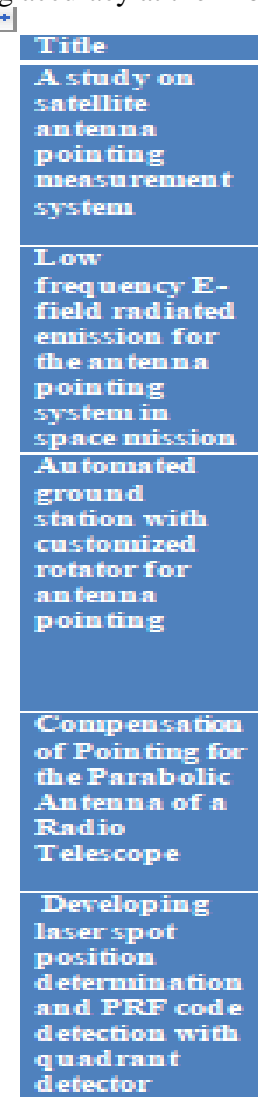

Xiao Wen, (2017) implement the model and measurement of the laser spot position with quadrant detector based on the Pulse Repetition Frequency (PRF) with two microcontroller(MUC1, MUC2) ATmega32 and semi-laser weapon, MUC1 generate laser pulse toward target MUC2 decode the reflected pulse through the quadrant detector, ATmega32 microcontroller get the output from the ADC and PRF and perform the processing on signal to evaluate position at low cost.

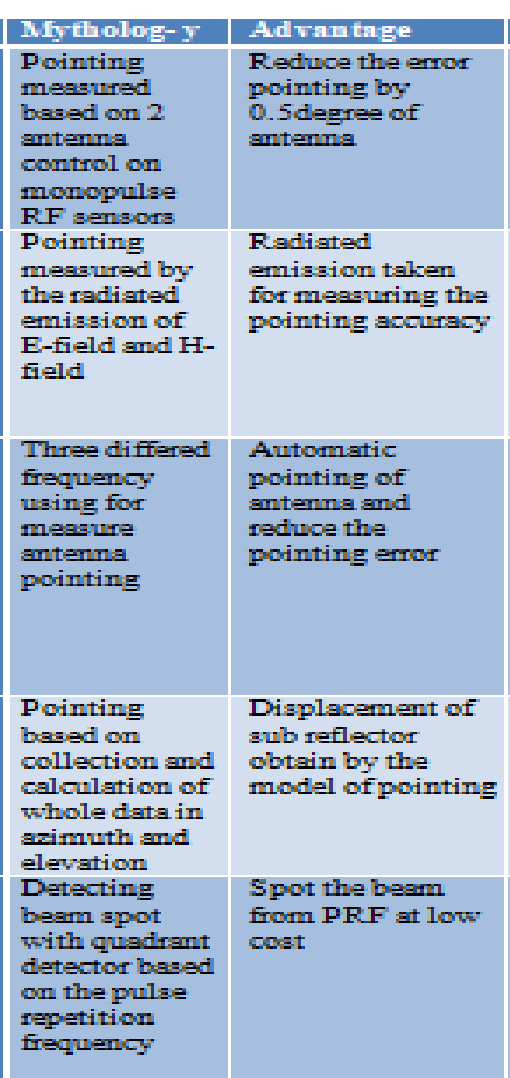

Fig. 1: Comparative Study

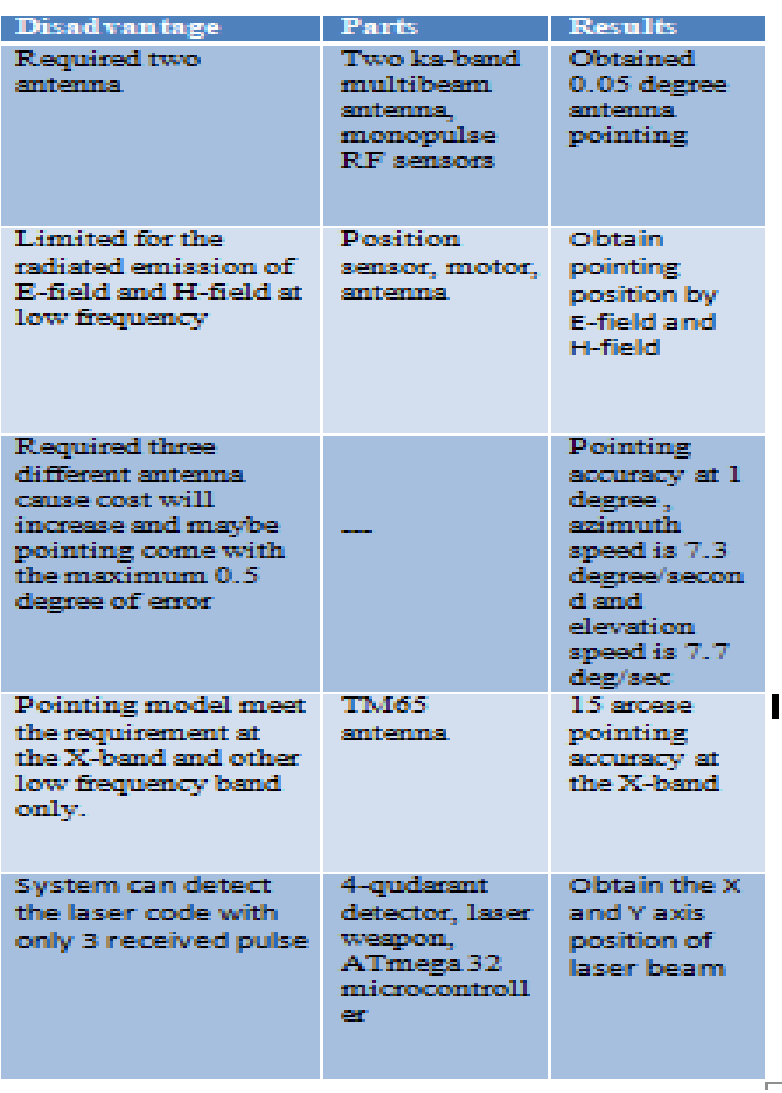

James S, (2009) analysing control problem of laser beams aiming in dynamic disturbances condition, using light intensity for feedback. The beam is conducted with a bi-axial MEMS mirror, which is steered by a control signal which is generated by sense of processing beam intensity by a single photodiode. Since the pointing location of laser beam is not be assume to available for real-time control, a static nonlinear mapping from the twodimensional beam location to the sensor measurement is estimated with the use of the least-squares algorithm, using data from an optical position sensor (OPS).

\section{Algorithm}

\section{A. Antenna Pointing Model}

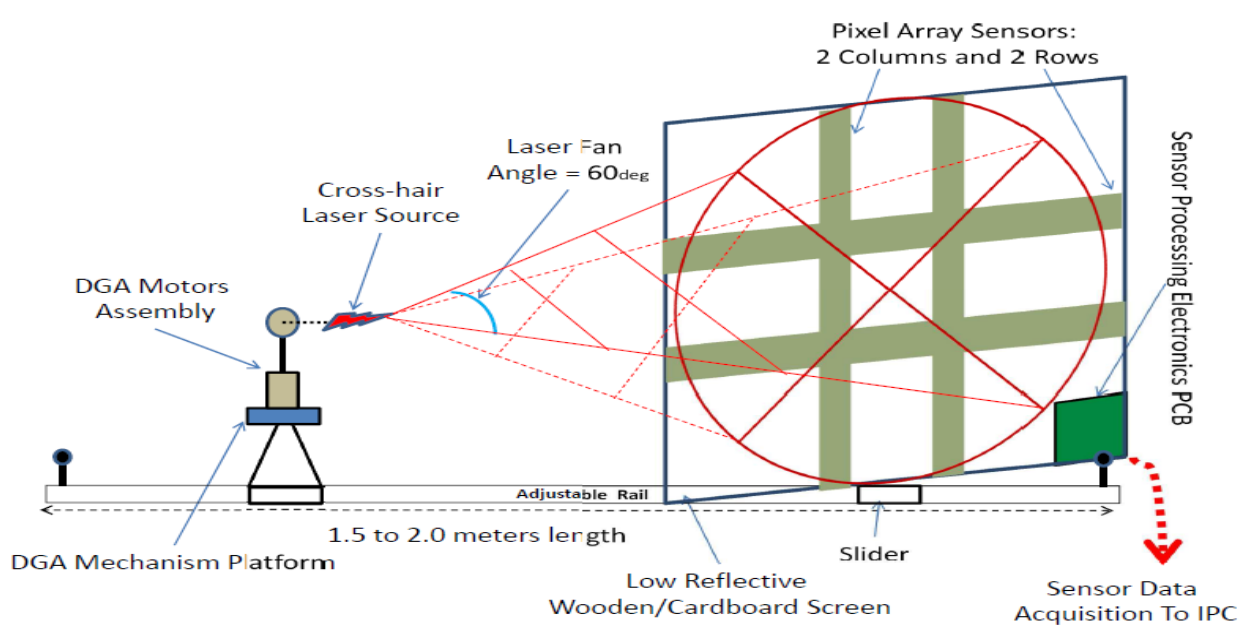

Fig. 2: Antenna Pointing System Setup 
Implementing a model for pointing of antenna based on laser weapon and linear array sensors setup is shown in Fig.2. This model mainly consists of a antenna, rotator system, one crosshair laser weapon, linear pixel array sensors (TSL1410R2), a laser fun, sensor processing electronics PCB, FPGA fabric, 32-bit ARM cortex M3 processor.

A crosshair laser weapon is most important component for measuring the pointing of antenna which is mounted on the antenna system, that weapon is intended to emitting the laser beam on the sensor board at cross pattern with 60 degree and the sensor board are consist of 40 pixel array sensors which is used to detect the beam position by the 32-bit processor and send the command to the rotator system, which is responsible for rotate the antenna in desire direction, for that here antenna system is assembled with the rotator system for easy operate.

\section{B. Reading of TSL1410}

In order to obtain the antenna position, it is necessary to get the accurate data from the sensors about light energy.

TSL1410 is consist of 1280 pixel, also called photodiode, each one have integration circuitry and a sample capacitor is connected at the output of the integration circuit in order to accumulated the charge.

Photodiode generate the photo-current when the light energy is impinging on the pixel that is then integrate by the integration circuit of associate pixel. During the integration period the sample capacitor is connect with the output of integration circuit associated by each pixel and charge is store in capacitor is directly proportion to the intensity of light.

The output of this sensor is initiate by internal logic that required only clock and serial input (SI) signal.An output cycle is initiated by clocking in a logic 1 on SI, the charged accumulated by the sample capacitor is sequentially connected to the output amplifier that is responsible for generate a voltage on analog output (AO).

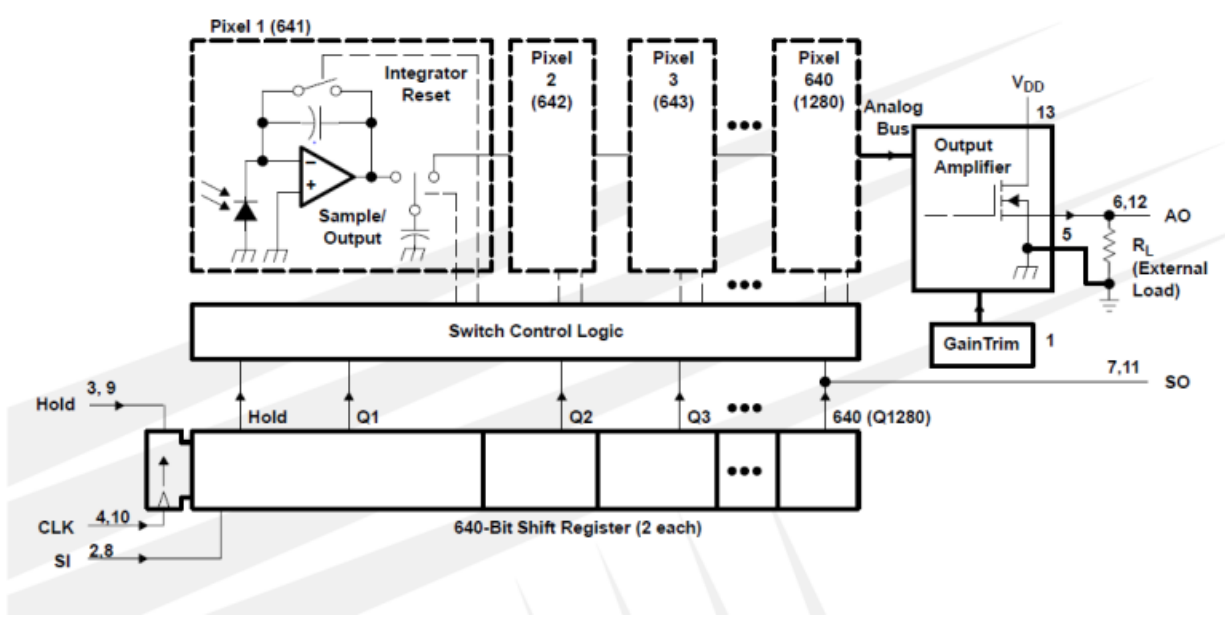

Fig. 3: Functional block diagram of liner pixel sensor connected in series.

For reading the analog out from the sensor, using a Arduino board. The TSL1410 is belonging to a linear sensor array family. If TSL1410 read output serially needs to be connected with 3 logical cord or need to be connected with 4 logical cord for parallel read of output.

The logical cords to the TSL1402R are clock (CLK), Serial Input (SI) and (1 or 2) Analog Output (AO). In addition, there are the cords for $+5 \mathrm{~V}$ and GND.

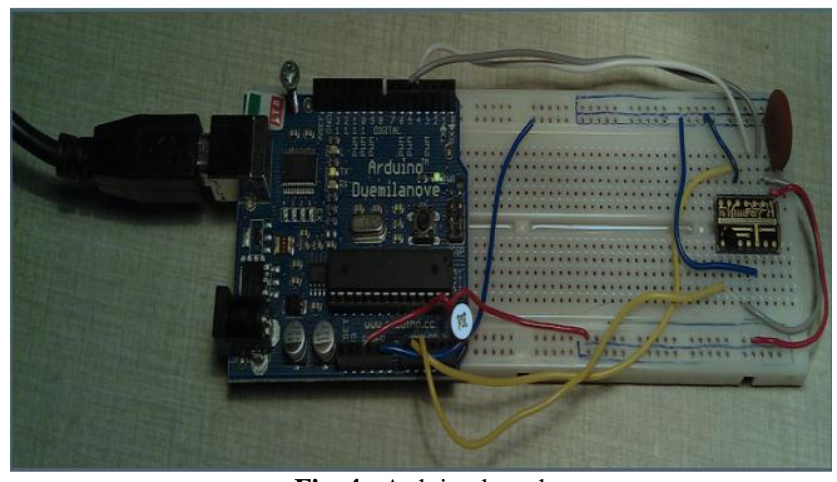

Fig. 4: Arduino board

Arduino board is enough for reading only one sensor but here we are using array of 40 pixel sensors shown in figure 4 . For reading the analog output from the array of 40 sensors required a processor board that have more number of IO to capable take the analog data, therefore we are using FPGA board, which is received the analog data from the pixel array sensor and find the activated sensor s.
Lesar light is impinging on the sensore board which is give the analog data of array of pixel sensors which is received by the FPGA board and gives information about the active sensors. We need to store this sensor data in file for mapping the pixel in order to find out the intercept point of laser beam.

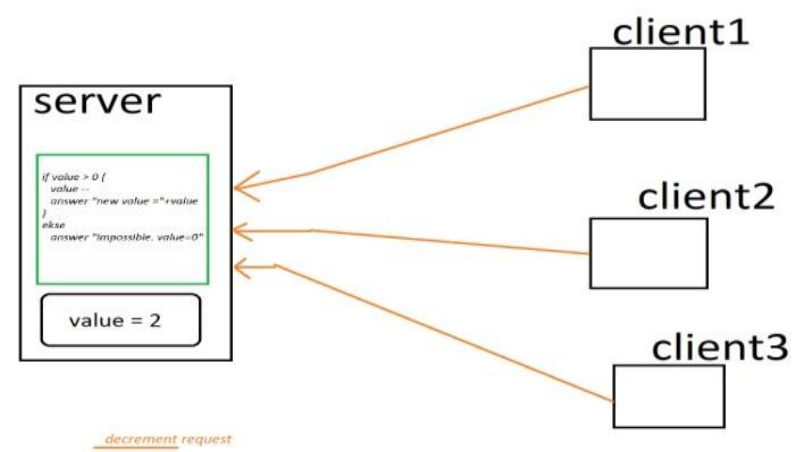

Fig.5: client server

Creating a client/server program in java for storing sensor data in file, where processing board perform a server and sensor board performed client and it will be use also for mapping of pixels.

\section{Reading Sensor Data}

Reading of sensors data is possible only when clock is available and after SI and sensor out on signals generated.

\section{Data storage}




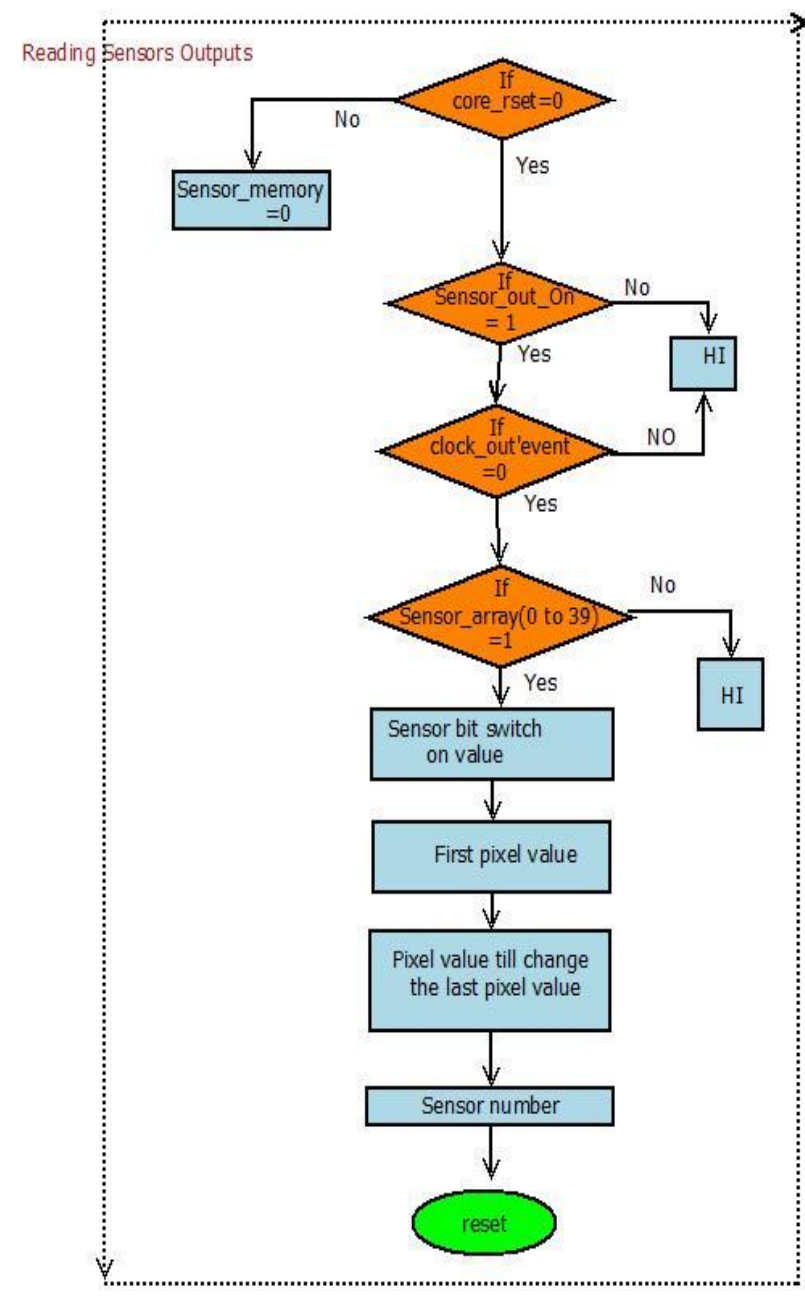

Fig.6: Sensor data reading flow chart

In every step controller check core reset, same here controller also check reset signal is reset signal is active then sensor memory will be empty but when core reset is not active i.e. core_rest $=1$ then controller concentrate on eventing 0 on clock out, after that if any sensors controller check which light sensor goes active. Given the pixel first bit switch on, pixel first bit switch ON value we can take according to clock and pixel value till last pixel and the sensor number which give the information about the how many sensors goes active. Figure 6 shows the details flow chart for reading sensor data.

Sensor board consists of array of 40 linear sensors ( 2 rows and 2 columns) and power supply by external $3.3 \mathrm{~V}$ DC voltage source.Arduino board is enough for reading only few sensors but for reading all 40 array sensors required large board who have capacity to take all data from the sensors are using a Smart-Fusion Board that is fusion of FPGA and Microcontroller shown in figure 7.

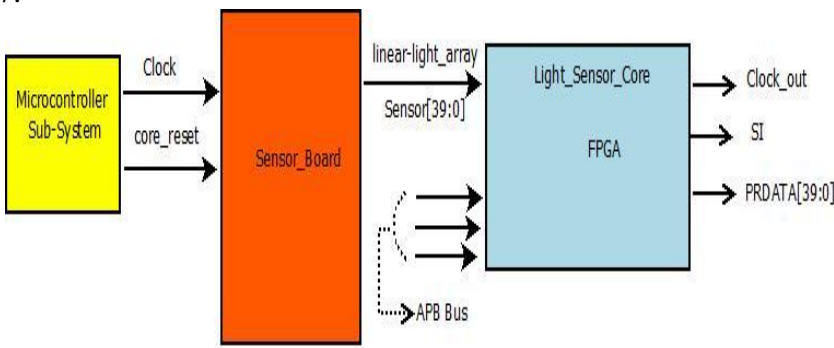

Fig.7: Block diagram of interface sensor with microcontroller and FPGA

\section{Results and Discussion}

When SI clocking in (SI = 1) at fabric clock 2 and SI become 0 when fabric clock 3 and Si counter start after immediately of SI become high and $\mathrm{Si}$ _counter run till 4000 after that process will repeat. No data coming out in first SI, PRDATA is 0x00 can be observed in figure 8 simulation 1 result output. After 4000 of si_counter si again goes high and again si_counter run till 4000 count.

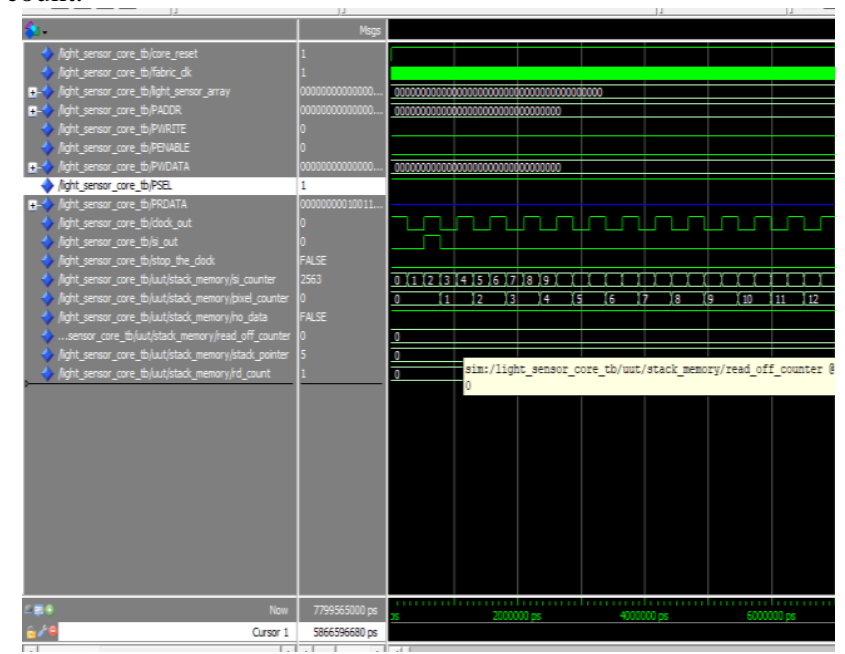

Fig.8: Simulation 1

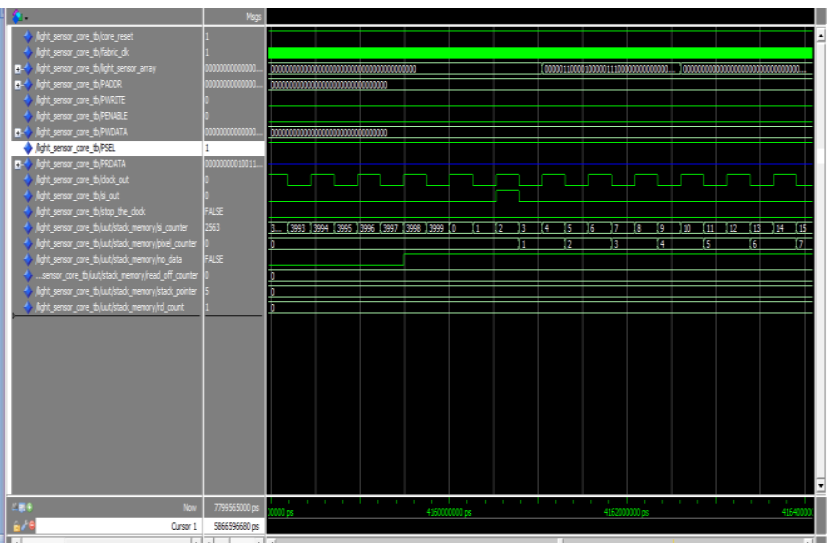

Fig.9: Simulation 2

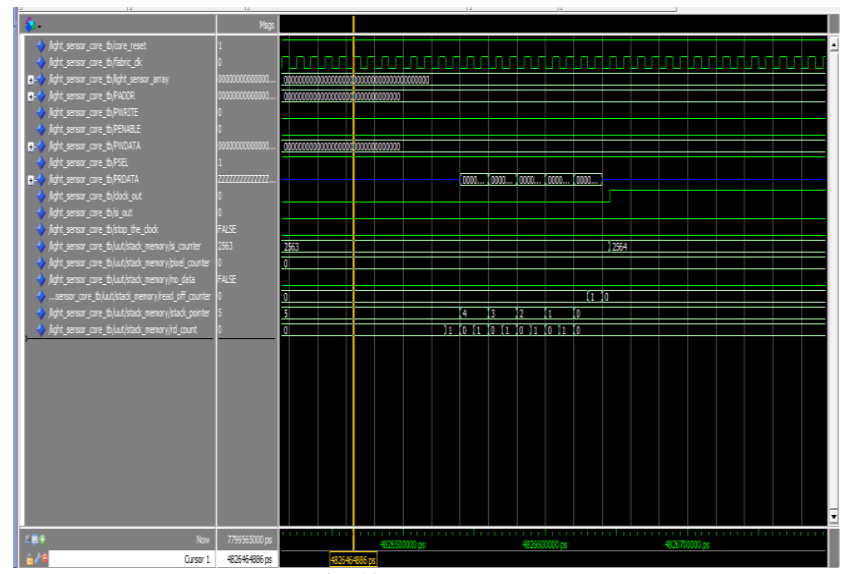

Fig.10: Simulation 3

Figure 10 shows the simulation 3 results which clearly indicates the few data coming out at stack pointer 4 .

\section{Conclusion and Future Work}

There has been extensive research work going on in space industry to pointing the antenna mechanism with minimum pointing error. Comparative observation towards algorithms on pointing the antenna mechanism like APM, APME, quadrant detector, PRF has been carried out. In my future work I want to propose a low cost 
pointing measurement system with a minimum pointing error solution for ground antenna test system.

\section{References}

[1] Hernandez, A. M., Escolar, J. G., Trillo, A. A., \& Pardo, J. F. (2017, September). Low frequency electric field radiated emissions for the antenna pointing subsystems in space missions. In Electromagnetic Compatibility-EMC EUROPE, 2017 International Symposium on (pp. 1-6). IEEE.

[2] Anggara, R. S., Wijanto, H., Prasetyo, A. D., \& Syihabuddin, B. (2014, November). Automated ground station with customized rotator for antenna pointing using compass sensor. In Electrical Engineering and Computer Science (ICEECS), 2014 International Conference on (pp. 59-64). IEEE.

[3] Yu, H. (2017, May). Compensation of pointing for the parabolic antenna of a radio telescope. In Progress In Electromagnetics Research Symposium-Spring (PIERS), 2017 (pp. 190-194). IEEE.

[4] Heweage, M. F., Wen, X., Mokhtar, A., \& Eldamarawy, A. (2017). Developing Laser Spot Position Determination and PRF Code Detection with Quadrant Detector. World Academy of Science, Engineering and Technology, International Journal of Electrical and Computer Engineering, 4(12).

[5] Pérez-Arancibia, N. O., Gibson, J. S., \& Tsao, T. C. (2009, June) Laser beam pointing and stabilization by intensity feedback control In American Control Conference, 2009. ACC'09. (pp. 2837-2842). IEEE.

[6] Bauer, F., Danielyan, V., Horvat, S., \& Kroha, H. (2004). Studies of semitransparent optoelectronic position sensors. IEEE Sensors Journal, 4(3), 329-336.

[7] Githens, J. A., Kelly, H. P., Lozier, J. C., \& Lundstrom, A. A. (1963). Antenna pointing system: organization and performance. Bell Labs Technical Journal, 42(4), 1213-1221.

[8] Mishra, P. K., Nagesh, S. K., \& Govinda, K. V. (2011, December). Challenges in multibeam onboard Tracking Antenna charecterization: An ISRO experience. In Antenna Week (IAW), 2011 Indian (pp. 1-4). IEEE.

[9] Sundari, S. M. (2013, December). Design of Antenna Pointing System (APS)-its control and communication challenges. In Contro Communication and Computing (ICCC), 2013 Internationa Conference on (pp. 490-495). IEEE.

[10] Danielyan, V., Horvat, S., \& Kroha, H. (2002). Studies of semitransparent optoelectronic position sensors. In Sensors, 2002 Proceedings of IEEE (Vol. 2, pp. 829-835) 